\title{
Anestesia Peridural Contínua com Ropivacaína a 0,2\% Associada a Anestesia Geral para Cirurgia do Abdômen Superior em Crianças *
}

\section{Continuous Epidural Anesthesia with 0.2\% Ropivacaine Associated to General Anesthesia for Upper Abdominal Surgery in Children}

Jyrson Guilherme Klamt, TSA ${ }^{1}$, Luis Vicente Garcia, TSA ${ }^{1}$, Renato Mestriner Stocche, TSA ${ }^{1}$, Marlene Paulino dos Reis, TSA ${ }^{2}$

\begin{abstract}
RESUMO
Klamt JG, Garcia LV, Stocche RM, Reis MP - Anestesia Peridural Contínua com Ropivacaína a 0,2\% Associada a Anestesia Geral para Cirurgia do Abdômen Superior em Crianças
\end{abstract}

\begin{abstract}
Justificativa e Objetivos - Técnicas anestésicas têm sido empregadas em pacientes pediátricos para vários tipos de cirurgias, apresentando entre outras vantagens, a analgesia pós-operatória. O objetivo deste estudo foi avaliar o ritmo de infusão de propofol e a recuperação pós-anestésica de crianças submetidas à cirurgia abdominal alta sob anestesia peridural torácica com ropivacaína a 0,2\%, associada à anestesia geral com propofol ou propofol mais sufentanil.
\end{abstract}

Método - Vinte e seis crianças ASA I, II e III, com idades entre 0 e 4 anos, submetidas à cirurgia abdominal alta foram selecionadas para anestesia peridural torácica $\left(T_{7}-T_{8}\right) \mathrm{com}$ ropivacaína a $0,2 \%\left(1,5 \mathrm{ml} . \mathrm{kg}^{-1}\right)$. Foram divididas aleatoriamente em dois grupos: Propofol (infusão de propofol) e Sufentanil (infusão de propofol mais sufentanil $1 \mu \mathrm{g} \cdot \mathrm{kg}^{-1}$ ). Os ritmos de infusões de propofol foram de 20 e $10 \mathrm{mg}^{\mathrm{kg}} \mathrm{kg}^{-1} \cdot \mathrm{h}^{-1}$ nos grupos Propofol e Sufentanil, respectivamente, ajustadas de modo a manter a pressão arterial cerca de $20 \%$ dos valores pré-indução e interrompidas 10 a 15 minutos antes do final estimado da cirurgia. A recuperação pós-anestésica foi avaliada através de uma escala modificada de Aldrete-Kroulik e a sedação avaliada através de uma escala de 5 pontos.

Resultados - Duas crianças de cada grupo foram excluídas por problemas técnicos. O ritmo de infusão foi significativamente menor no grupo Sufentanil em relação ao grupo Propofol durante 100 minutos após o início da cirurgia. Os tempos para extubação e transferência para a sala de recuperação pós-anestésica (SRPA) foram significativamente menores no grupo Propofol, porém a intensidade e a duração da sedação foram maiores nesse grupo em relação ao grupo Sufentanil. Os escores de recuperação foram similares nos dois grupos. Após 3 horas na SRPA, todos pacientes haviam atingido os critérios para transferência para as enfermarias. Hipotensão arterial transitória foi observada em 2 pacientes do grupo Sufentanil.

\footnotetext{
* Recebido da (Received from) Disciplina de Anestesiologia da Faculdade de Medicina de Ribeirão Preto (FMRP- USP), Ribeirão Preto, SP 1. Professor Doutor do Dept ${ }^{\circ}$ de Biomecânica, Medicina e Reabilitação do Aparelho Locomotor - Disciplina de Anestesiologia da FMRP - USP

2. Professora Associada do Dept ${ }^{\circ}$ Biomecânica, Medicina e Reabilitação do Aparelho Locomotor - Disciplina de Anestesiologia da FMRP - USP
}

Apresentado (Submitted) em 03 de abril de 2002

Aceito (Accepted) para publicação em 15 de agosto de 2002

Correspondência para (Mail to):

Dr. Jyrson Guilherme Klamt

Av. dos Bandeirantes, 3900 - Monte Alegre

14048-900 Ribeirão Preto, SP

E-mail: jgklamt@fmrp.usp.br

(c) Sociedade Brasileira de Anestesiologia, 2003
Conclusões - A combinação da anestesia peridural torácica contínua com ropivacaína a $0,2 \%\left(1,5 \mathrm{ml}^{\mathrm{kg}} \mathrm{kg}^{-1}\right)$ associada à infusão de propofol promove anestesia efetiva e segura para cirurgias abdominais altas em crianças. O ritmo de infusão de propofol e o tempo de sedação foram reduzidos com a adição de sufentanil.

UNITERMOS: ANESTESIA, Pediátrica; ANESTÉSICOS, Local: ropivacaína; TÉCNICAS ANESTÉSICAS, Regional: peridural contínua

\section{SUMMARY}

Klamt JG, Garcia LV, Stocche RM, Reis MP - Continuous Epidural Anesthesia with $0.2 \%$ Ropivacaine Associated to General Anesthesia for Upper Abdominal Surgery in Children

Background and Objectives - Several anesthetic techniques have been proposed for different pediatric surgeries to promote postoperative analgesia, among other advantages. This study aimed at evaluating propofol infusion rate and postanesthetic recovery of children submitted to upper abdominal surgeries under epidural anesthesia with $0.2 \%$ ropivacaine associated to general anesthesia with propofol or propofol plus sufentanil.

Methods - Participated in this study 26 children physical status ASA I, II and III, aged 0 to 4 years, were scheduled to upper abdominal surgeries under thoracic epidural anesthesia $\left(T_{7}-T_{8}\right)$ with $0.2 \%$ ropivacaine $\left(1.5 \mathrm{ml} . \mathrm{kg}^{-1}\right)$. They were randomly distributed in two groups: Propofol (propofol infusion) and Sufentanil (propofol infusion plus $1 \mu \mathrm{g} \cdot \mathrm{kg}^{-1}$ sufentanil). Propofol infusion rates were 20 and $10 \mathrm{mg} \cdot \mathrm{kg}^{-1} \cdot \mathrm{h}^{-1}$ for the Propofol and Sufentanil groups, respectively, adjusted to maintain blood pressure in approximately $20 \%$ of baseline values and withdrawn 10 to 15 minutes before estimated surgery completion. Postanesthetic recovery was evaluated by a modified Aldrete-Kroulik scale and sedation was evaluated by a 5 grade score.

Results - Techical difficulties excluded two children of each group. Infusion rate was significantly slower in the Sufentanil group as compared to the Propofol group during 100 minutes after beginning of surgery. Time for extubation and referral to post-anesthetic recovery unit (PACU) was significantly shorter for the Propofol group, however sedation intensity and duration were longer in this group as compared to Sufentanil group. Recovery scores were similar for both groups. Three hours after PACU admission all patients were meeting criteria to be transferred to the ward. Transient arterial hypotension was observed in 2 Sufentanil group patients.

Conclusions - Continuous thoracic epidural anesthesia with $0.2 \%$ ropivacaine $\left(1.5 \mathrm{mg} \cdot \mathrm{kg}^{-1}\right)$ associated to propofol infusion provides effective and safe anesthesia for upper pediatric abdominal surgeries. Propofol infusion rate and sedation duration were decreased with the association of sufentanil.

KEY WORDS: ANESTHESIA, Pediatric; ANESTHETICS: Local: ropivacaine: ANESTHETIC TECHNIQUES: Regional: continuous epidural 


\section{INTRODUÇÃO}

$\mathrm{N}_{\mathrm{c}}^{\mathrm{a}}$ nossa prática anestésica de rotina, a introdução de catéteres no espaço peridural torácico através de punção sacral, transacral ou lombar, tem-se mostrado uma técnica confiável e reprodutível, o que está de acordo com outros autores $^{1,2}$. Desde que o bloqueio sensorial atinja todos os segmentos envolvidos na região cirúrgica é possível manter anestesia geral superficial com rápida recuperação e excelente analgesia pós-operatória.

A ropivacaína, um anestésico local de longa duração, possui menor toxicidade cardíaca e no sistema nervoso central, é equipotente e promove maior separação entre os efeitos motores e sensoriais quando comparados a bupivacaína ${ }^{3,4}$. Na concentração de $0,2 \%$ promove analgesia per-operatória para cirurgia abdominal similar a bupivacaína a $0,25 \%$ após administração peridural em volumes iguais ${ }^{5}$. A ropivacaína possui propriedades vasoconstritoras que reduz o risco e a intensidade da absorção sistêmica quando comparada à bupivacaína e quando empregada sem vasoconstritor parece ser segura em doses de até $3,75 \mathrm{mg} \cdot \mathrm{kg}^{-1}$ 6,7.

O propofol é um agente venoso altamente apropriado e confiável para indução e manutenção da anestesia geral em crianças. Apresenta estabilidade hemodinâmica, especialmente sob anestesia regional ${ }^{8-10}$. A recuperação pós-anestésica é rápida e completa, com baixa incidência de náuseas e vômitos e de fácil controle de profundidade anestésica ${ }^{11-13}$. A concentração anestésica do propofol necessária para manter a estabilidade hemodinâmica e a imobilidade depende da intensidade do estimulo (intubação traqueal, incisão da pele e tração de vísceras e mesentério) e da associação de outras drogas anestésicas ${ }^{13-15}$.

O objetivo do presente estudo foi o de verificar a eficácia, segurança, necessidade do propofol e característica da recuperação, em crianças anestesiadas com infusão de propofol ou propofol mais sufentanil associadas a ropivacaína a $0,2 \%$ $\left(1,5 \mathrm{ml} . \mathrm{kg}^{-1}\right)$ por via peridural torácica e submetidas à cirurgias intra-abdominais e supra-umbilicais.

\section{MÉTODO}

Após a obtenção da aprovação do Conselho de Ética em Pesquisa do Hospital das Clínicas de Ribeirão Preto e obtenção de consentimento pós-informado dos pais, 26 crianças, estado físico ASA I, II e III, com idades entre 0 e 4 anos escaladas para cirurgia abdominal alta, foram aleatoriamente designadas para receber anestesia geral com infusão de propofol (grupo Propofol) ou infusão de propofol associado ao sufentanil (1 $\mu \mathrm{g} . \mathrm{kg}^{-1}$ ) (grupo Sufentanil). Ambos os grupos receberam anestesia peridural torácica com ropivacaína a $0,2 \%\left(1,5 \mathrm{ml} . \mathrm{kg}^{-1}\right)$. Foram excluídos do estudo pacientes com distúrbios de coagulação, anormalidade neurológica e doença cardiovascular.

Foram monitorizados a freqüência cardíaca, bulhas cardíacas, pressões arteriais diastólica e sistólica não invasivas, temperatura central (nasofaringeal), $\mathrm{SpO}_{2}, a \mathrm{P}_{\mathrm{ET}} \mathrm{CO}_{2}$ e a transmissão neuromuscular (seqüência de quatro estímu- los). Todos os pacientes foram hidratados utilizando-se solução de Ringer com lactato, 10 a $15 \mathrm{ml} \cdot \mathrm{kg}^{-1} \cdot \mathrm{h}^{-1}$, e solução glicosada a $10 \%$ foi administrada a um ritmo de $5 \mathrm{mg} \cdot \mathrm{kg}^{-1} \cdot \mathrm{min}^{-1}$. A anestesia geral foi induzida com propofol ( 3 a $5 \mathrm{mg} \cdot \mathrm{kg}^{-1}$ ), precedida por lidocaína $\left(1,5 \mathrm{mg} \cdot \mathrm{kg}^{-1}\right)$, por via venosa. O propofol a $1 \%$ foi injetado lentamente (aproximadamente a 0,1 $\mathrm{ml} . \mathrm{kg}^{-1} \cdot \mathrm{min}^{-1}$ ) até a perda do reflexo palpebral. O relaxamento muscular foi obtido com atracúrio $\left(0,5 \mathrm{mg} \cdot \mathrm{kg}^{-1}\right)$. Doses adicionais de atracúrio $\left(0,1 \mathrm{mg} \cdot \mathrm{kg}^{-1}\right)$ foram administradas, se necessárias.

Após a indução anestésica, seguiu-se a colocação de cateter peridural torácico. A punção peridural foi realizada com agulha $18 \mathrm{G}$ entre $L_{5}-T_{12}$ nas crianças acima de 3 meses ou com peso corporal maior que $7 \mathrm{~kg}$ ou por via caudal e trans-sacral nas demais crianças. Um cateter (20G) foi introduzido no espaço peridural na direção cefálica, em distância calculada, para que a ponta atingisse a região torácica $\left(T_{7}-T_{8}\right)$. A ropivacaína foi injetada pelo cateter na velocidade aproximada de $0,2 \mathrm{ml} \cdot \mathrm{kg}^{-1} \cdot \mathrm{min}^{-1}$ com o paciente em decúbito dorsal, cerca de 30 graus de céfalo-declive. Aos primeiros $0,2 \mathrm{ml} . \mathrm{kg}^{-1}$ de solução de ropivacaína, foi adicionada adrenalina (1:200.000), para dose teste.

A anestesia geral foi mantida com propofol 20 e 10 $\mathrm{mg} \cdot \mathrm{kg}^{-1} \cdot \mathrm{h}^{-1}$ nos grupos Propofol e Sufentanil, respectivamente. O ritmo de infusão foi alterado a cada 5 minutos de modo a manter a pressão arterial em torno de $20 \%$ dos valores pré-indução. Bolus de 0,5 a $1 \mathrm{mg} \cdot \mathrm{kg}^{-1}$ de propofol seriam administrados em caso de anestesia inadequada (aumento do tônus muscular, pressão arterial, freqüência cardíaca e respiratória). A infusão de propofol foi interrompida 10 a 15 minutos antes do término estimado da cirurgia.

Os pacientes foram ventilados mecanicamente para manter a $\mathrm{P}_{\mathrm{ET}} \mathrm{CO}_{2}$ entre 30 e $35 \mathrm{mmHg}$ e $\mathrm{SpO}_{2}$ maior que $95 \%$ usando uma mistura de ar e oxigênio através do sistema de Bain. Após o fechamento da aponeurose posterior, a ventilação espontânea seria permitida se o paciente pudesse manter a $\mathrm{P}_{\mathrm{ET}} \mathrm{CO}_{2}$ menor que $55 \mathrm{mmHg}$ e a $\mathrm{SpO}_{2}$ maior que $95 \% \mathrm{com}$ um fluxo de gás de $170 \mathrm{ml} \cdot \mathrm{kg}^{-1} \cdot \mathrm{min}^{-1}$ e CPAP de 3 a $5 \mathrm{cmH}_{2} \mathrm{O}$ através do sistema de Jackson-Rees. O bloqueio neuromuscular residual foi antagonizado ao final da cirurgia com atropina $\left(25 \mu \mathrm{g} \cdot \mathrm{kg}^{-1}\right)$ e neostigmina $\left(50 \mu \mathrm{g} \cdot \mathrm{kg}^{-1}\right)$. A extubação foi realizada na sala de cirurgia quando os pacientes reagiam intensamente ao tubo traqueal, exibiam padrão respiratório normal, abriam os olhos e moviam os 4 membros. Naloxona $\left(5 \mathrm{a} 10 \mu \mathrm{g} \cdot \mathrm{kg}^{-1}\right)$ por via venosa seria administrada, se os pacientes apresentassem depressão respiratória (freqüência respiratória menor que $14 \mathrm{rpm}$ ) e miose após 5 minutos do término da cirurgia.

Os pacientes foram transferidos para a SRPA quando atingiam um escore $\geq 8$ na escala de recuperação pós-anestésica de Aldrete-Kroulik modificada ${ }^{16}$ e um escore $\geq 3$ em escala de sedação de 5 pontos ${ }^{17}$. Na SRPA, a sedação e a recuperação anestésica, a pressão arterial e a freqüência respiratória foram medidas após 15, 30 e 60 minutos e, a seguir, a cada hora. $\mathrm{A} \mathrm{SpO}_{2}$ e a freqüência cardíaca foram monitorizadas continuamente. Ador foi tratada com morfina $\left(30 \mu \mathrm{g} . \mathrm{kg}^{-1}\right)$ por via peridural mais cetroprofeno $\left(2 \mathrm{mg} \cdot \mathrm{kg}^{-1}\right.$ por via venosa, 
cada 8 horas) e tramadol ( $\left.1 \mathrm{mg} \cdot \mathrm{kg}^{-1}\right)$, por via venosa, empregado como analgesia, quando os pacientes exibissem escore de 3 em uma escala de dor de 3 pontos ( $1=$ nenhuma dor ou dormindo; 2 = dor fraca (choro passível de ser confortado); 3 = dor moderada a grave, requer analgésico (choro ou agitação que não pode ser confortado, ou queixa de dor, ou pais acreditam que a criança está com dor). Complicações tais como depressão respiratória ( $F R<14$ rpm), náuseas e vômitos foram registrados durante 24 horas.

Os dados não paramétricos dos grupos foram analisados pelos testes de Mann-Whitney e dados de freqüência pelo teste de Qui-quadrado. Foi considerado significante valor de $p<$ 0,05 .

\section{RESULTADOS}

Vinte e seis pacientes foram selecionados para o estudo, mas 2 pacientes foram excluídos em cada grupo devido à elevada resistência à injeção pelo cateter peridural.

Não houve diferença significativa nas variáveis demográficas e duração da cirurgia entre os dois grupos (Tabela I).

Tabela I - Dados Demográficos, Duração da Cirurgia e Local de Punção

\begin{tabular}{lcc}
\hline & $\begin{array}{c}\text { Sufentanil } \\
(\mathrm{n}=11)\end{array}$ & $\begin{array}{c}\text { Propofol } \\
(\mathrm{n}=11)\end{array}$ \\
\hline Idade (meses) * & $14 \pm 13,8$ & $13,7 \pm 12,6$ \\
Peso $(\mathrm{kg})^{*}$ & $7,7 \pm 8,2$ & $5,8 \pm 6,3$ \\
Sexo (M/F) & $7 / 4$ & $5 / 6$ \\
Duração da cirurgia (min) * & $103,7 \pm 14$ & $112,4 \pm 22,3$ \\
Local da punção (n de casos) & & 8 \\
$\quad$ Lombar & 7 & 3 \\
\hline Caudal & 4 & \\
\hline
\end{tabular}

* Valores expressos pela Média \pm DP

As doses de indução de propofol foram 4,1 $\pm 0,6$ e 4,5 \pm 0,7 $\mathrm{mg} . \mathrm{kg}^{-1}$ nos grupos Propofol e Sufentanil, respectivamente. O ritmo de infusão de propofol foi significativamente menor no grupo Sufentanil durante um período de 100 minutos (fase visceral da cirurgia) após o início da cirurgia (Figura 1). A pressão arterial sistólica foi mantida em torno de $20 \%$ dos valores imediatamente antes da indução da anestesia geral nos dois grupos; no entanto, a freqüência cardíaca aumentou acima de $20 \%$ nos pacientes do grupo Propofol e permaneceu constante ou tendeu a diminuir nos pacientes do grupo Sufentanil. Significativamente, mais pacientes do grupo Propofol receberam bolus durante a fase visceral da cirurgia (6 e 1 pacientes dos grupos Propofol e Sufentanil, respectivamente), particularmente quando o estômago e o esôfago eram retraídos. Os pacientes dos dois grupos também necessitaram 1 a 2 bolus antes do final da cirurgia para inibir os movimentos da cabeça durante a manipulação da sonda nasogástrica e do tubo traqueal. Nenhuma dose adicional de atracúrio foi necessária em qualquer paciente. Dois pacientes do grupo Sufentanil desenvolveram hipotensão arterial

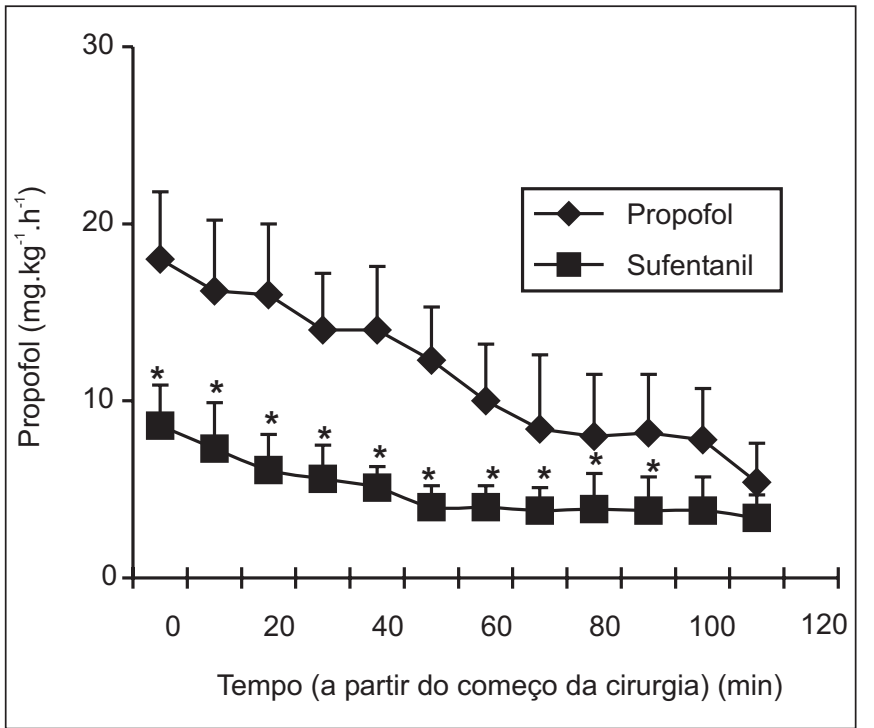

Figura 1 - Taxa de Infusão de Propofol (Média \pm DP)
$* p<0,05$

transitória, havendo retorno aos valores normais após 5 minutos da interrupção da infusão propofol.

A ventilação espontânea após o fechamento da aponeurose posterior foi possível em todos os pacientes do grupo Propofol e somente em 1 paciente do grupo Sufentanil. Todos os pacientes foram extubados na sala de cirurgia. Tempo significativamente maior foi necessário para extubação e transferência para a SRPA nos pacientes do grupo Sufentanil em relação aos pacientes do grupo Propofol (Figura 2). Exceto pelo grau de sedação, que foi significativamente mais profunda e prolongada no grupo Propofol, as demais características de recuperação foram similares nos 2 grupos (Figura 3). Naloxona foi administrada em 5 pacientes do grupo Sufentanil. Todos os pacientes atingiram os critérios de alta (escore de recuperação $\geq 8$, escore de sedação $\geq 3$, ausência de

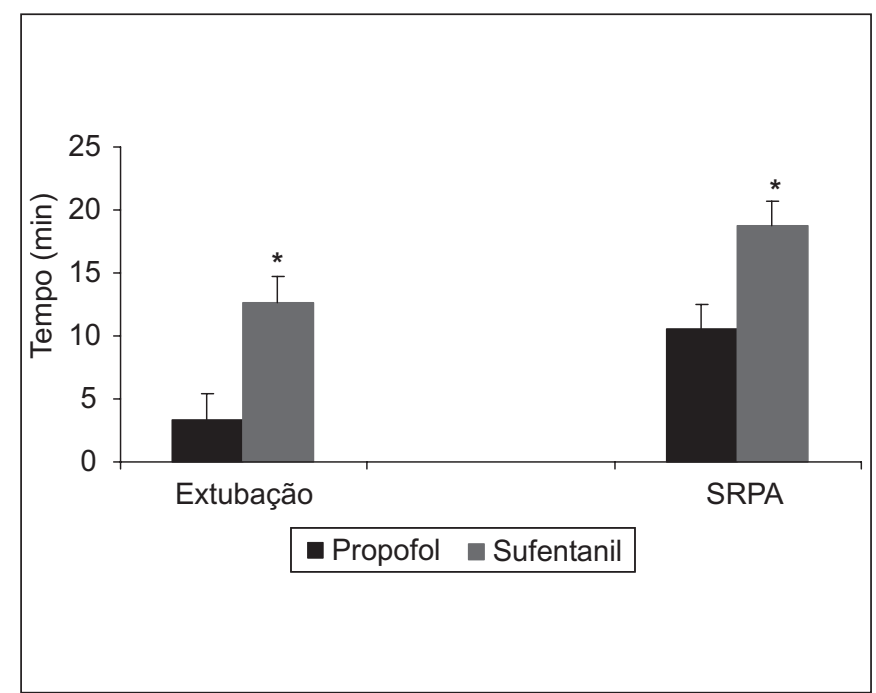

Figura 2 - Tempo para Extubação e Transferência para a SRPA após o Final da Cirurgia (Média \pm DP)

Revista Brasileira de Anestesiologia Vol. 53, N² 2, Março - Abril, 2003 


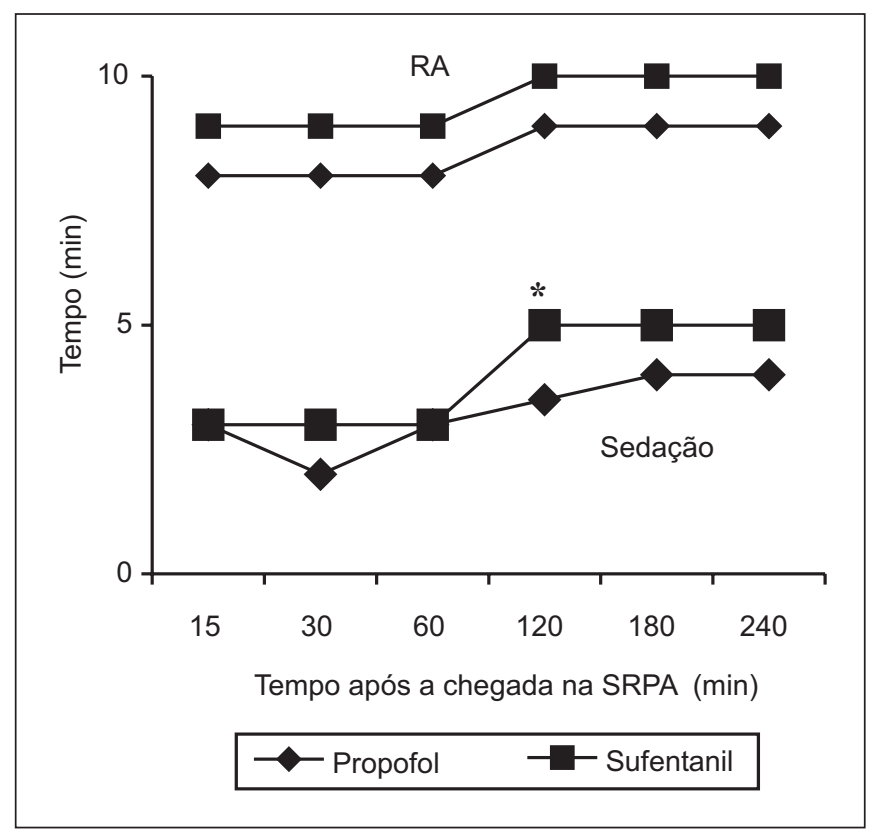

Figura 3 - Recuperação Anestésica e Sedação na SRPA (Média \pm DP)

$* p<0,05$

queixas, dor ou comportamento sugestivo de dor, náuseas, vômitos e sinais de bloqueio motor) após 3 horas da chegada na SRPA. Hipotensão arterial, bradicardia, disritmia cardíaca, obstrução das vias aéreas, depressão respiratória, ou sinais de bloqueio motor dos membros inferiores ou superiores não foram observados nos pacientes dos dois grupos. Não foi necessária a administração de analgésicos a nenhum paciente dos dois grupos durante permanência na SRPA.

\section{DISCUSSÃO}

Ambas as técnicas utilizadas neste estudo podem ser consideradas anestesia venosa total, uma vez que após a injeção peridural de ropivacaína somente anestésicos venosos foram utilizados. A adição de sufentanil à infusão de propofol retardou a extubação e a transferência para a SRPA. Entretanto permitiu recuperação mais rápida, sobretudo devido à possibilidade de utilizar menor ritmo de infusão de propofol. Não foram observados obstrução das vias aéreas, laringoespasmo, depressão cardiorrespiratória, vômitos e bloqueio motor no período pós-operatório imediato.

As taxas de infusão de propofol necessárias no presente estudo estão próximas às recomendadas por Mc Farlan e col. ${ }^{18}$, os quais são aplicáveis a crianças entre 3 e 11 anos, mas não a neonatos e lactentes, os quais necessitam de doses ainda maiores. O ritmo elevado de infusão de propofol usado foi necessário para controlar a resposta hemodinâmica a procedimentos invasivos tais como intubação traqueal, punção peridural e canulação de veia central. Esse estudo demonstrou que a taxa de infusão pode ser reduzida pelo menos em 50\% com o emprego constante de sufentanil (1 $\left.\mu \mathrm{g} . \mathrm{kg}^{-1}\right)$. Baseado nas necessidades de propofol e fentanil, outro estudo concluiu que a tração abdominal e a incisão do peritônio são os estímulos mais intensos durante a cirurgia ${ }^{15}$, possivelmente o mesmo possa ocorrer para a tração do estômago e o diafragma. Houve apenas dois casos de hipotensão arterial transitórias. Nosso estudo é coerente com a observação da qual o propofol é bem tolerado por crianças com função cardíaca preservada, e que a pressão arterial somente diminui durante a indução, fato que é mais freqüente em crianças maiores e adolescentes. Da mesma forma, no nosso estudo, não foram registradas reduções clinicamente importantes da freqüência cardíaca e alterações do ritmo cardíaco com a associação de sufentanil ${ }^{10,19}$.

A recuperação rápida após infusão prolongada de propofol, geralmente descrita ${ }^{20}$, foi possível nos pacientes que receberam sufentanil. Entretanto, ocorreu dificuldade em assumir a ventilação espontânea em 5 pacientes do grupo Sufentanil, necessitando reversão com naloxona. Conseqüentemente, o tempo para extubação e encaminhamento para a SRPA foi maior no grupo Sufentanil. Sedação prolongada no grupo Propofol certamente é resultado da infusão prolongada e em taxa maior que a apresentada no grupo Sufentanil. Os níveis de anestésicos locais no plasma e seus efeitos sistêmicos estão diretamente relacionados às doses, independente dos volumes das soluções ${ }^{21}$. Para anestesia peridural caudal ou lombar, as doses recomendadas de bupivacaína variam de 2,5 a $5 \mathrm{mg} \cdot \mathrm{kg}^{-1}$, e as doses mais altas devem ser empregadas com a adição a adrenalina à solução de anestésico local ${ }^{21,22}$. Empregamos ropivacaína (3 $\left.\mathrm{mg} \mathrm{kg}^{-1}\right)$, e os primeiros $0,2 \mathrm{ml} . \mathrm{kg}^{-1}$ foram administrados com adrenalina (5 $\mu \mathrm{g} \cdot \mathrm{kg}^{-1}$ ) para permitir detecção de injeção intravascular acidental. A solução de ropivacaína a $0,2 \%$ foi injetada no espaço peridural pelo cateter na velocidade de $0,2 \mathrm{ml} \cdot \mathrm{kg}^{-1} \cdot \mathrm{min}^{-1}$ com objetivo de evitar aumento abrupto de pressão no espaço peridural. Considerando que a ropivacaína tem toxicidade sistêmica e absorção sistêmica mais lenta (propriedade vasoconstritora) do que a bupivacaína e um estudo experimental mostrou que em ratos jovens ocorre maior resistência à toxicidade de ropivacaína que em ratos adultos ${ }^{17,23}$, doses maiores que ropivacaína em relação à bupivacaína podem ser usadas. Conseqüentemente, bloqueio peridural mais extenso e prolongado pode ser atingido com segurança. Não obstante, uma série de fatores aumentam os riscos de toxidade sistêmica aos anestésicos locais em crianças em relação aos adultos ${ }^{24}$. A ropivacaína na dose de 3,75 $\mathrm{mg} \cdot \mathrm{kg}^{-1}$ apresenta pico de concentração plasmática ( $\mathrm{T}_{\mathrm{MAX}}$ ) cerca de 30 minutos após a administração caudal e mantém-se estável durante 3 horas ${ }^{7}$. A concentração plasmática mais elevada medida foi de 2,2 $\mu$ g e nenhum efeito neurológico foi observado. No nosso estudo, nenhum caso de toxicidade cardíaca ou neurológica foi observado. Para a bupivacaína, acredita-se que a concentração plasmática de 2 a 3 $\mu \mathrm{g} . \mathrm{ml}^{-1}$ seja o limiar para toxicidade do sistema nervoso central (convulsão) ${ }^{7,25}$. Deve-se reconhecer, no entanto, que o risco real de ocorrência de eventos adversos neurológicos ou outros efeitos adversos possíveis podem ter sido mascarados pela alta taxa de infusão de propofol e seus efeitos prolongados no período pós-operatório. 
Não foram observados sinais de bloqueio motor dos membros superiores, o que está em acordo com o perfil de bloqueio sensorial/motor descritos por König e col. ${ }^{7}$. A despeito do alto volume $\left(1,5 \mathrm{ml}^{\mathrm{kg}}{ }^{-1}\right)$ injetado no espaço peridural torácico, nenhuma criança desenvolveu hipotensão arterial (associada ao bloqueio peridural), bradicardia ou síndrome de Horner. Vários estudos estabeleceram a marcada estabilidade hemodinâmica quando se associa bloqueio peridural à anestesia geral em crianças com idades inferiores a 8 anos, sobretudo até aos 4 anos. Aextubação precoce e a baixa incidência de eventos adversos na recuperação anestésica também são características da associação do bloqueio peridural e anestesia geral em crianças ${ }^{1,2}$.

Em conclusão, nesse estudo, a anestesia peridural torácica com ropivacaína a $0,2 \%\left(1,5 \mathrm{ml} . \mathrm{kg}^{-1}\right)$ associada à anestesia geral com propofol ou propofol mais sufentanil promoveu anestesia segura. A associação de sufentanil diminuiu consideravelmente o consumo de propofol e o grau de sedação na SRPA. Contudo, pode prolongar o tempo para assumir respiração espontânea e, conseqüentemente, o tempo para extubação e encaminhamento para a SRPA.

\section{Continuous Epidural Anesthesia with $0.2 \%$ Ropivacaine Associated to General Anesthesia for Upper Abdominal Surgery in Children}

Jyrson Guilherme Klamt, TSA, M.D., Luis Vicente Garcia, TSA, M.D., Renato Mestriner Stocche, TSA, M.D., Marlene Paulino dos Reis, TSA, M.D.

\section{INTRODUCTION}

According to our daily practice, the introduction of catheters in the thoracic epidural space through sacral, transacral or lumbar puncture, has been proven a reproducible and reliable technique, which is in line with the literature ${ }^{1,2}$. Provided sensory block reaches all segments involved in the surgical region, it is possible to maintain superficial general anesthesia with fast recovery and excellent postoperative analgesia.

Ropivacaine, a long lasting local anesthetic, induces less cardiac and central nervous system toxicity, is equipotent and promotes a better split between motor and sensory effects as compared to bupivacaine ${ }^{3,4}$. In the concentration of $0.2 \%$, it promotes perioperative analgesia for abdominal surgeries similar to $0.25 \%$ bupivacaine after epidural administration in equal volumes ${ }^{5}$. Ropivacaine has vasoconstriction properties which decrease risk and systemic absorption intensity as compared to ropivacaine and, when used without vasoconstrictor, seems to be safe in doses up to $3.75 \mathrm{mg} \cdot \mathrm{kg}^{-16,7}$.
Propofol is a highly adequate and reliable venous agent for induction and maintenance of general anesthesia in children. It has hemodynamic stability, especially under regional anesthesia ${ }^{8-10}$. Post-anesthetic recovery is fast and complete with low incidence of nausea and vomiting and easy control of anesthetic depth ${ }^{11-13}$. Propofol anesthetic concentration needed to maintain hemodynamic stability and immobility depends on stimulation intensity (tracheal intubation, skin incision and visceral and mesentery traction) and on the association of other anesthetic drugs ${ }^{13-15}$.

This study aimed at evaluating the efficacy, safety and propofol need, in addition to recovery characteristics in anesthetized children with propofol infusion or propofol plus sufentanil associated to epidural thoracic $0.2 \%$ ropivacaine $\left(1.5 \mathrm{ml} . \mathrm{kg}^{-1}\right)$ submitted to supra-umbilical intra-abdominal surgeries.

\section{METHODS}

After the Research Ethical Committee, Hospital das Clinicas, Ribeirão Preto, approval and the informed consent of parents, 26 children physical status ASA I, II and III, aged 0 to 4 years and scheduled for upper abdominal surgery were randomly assigned to receive general anesthesia with propofol infusion (Propofol group) or propofol infusion associated to 1 $\mu \mathrm{g} . \mathrm{kg}^{-1}$ sufentanil (Sufentanil group). Both groups received epidural thoracic anesthesia with $0.2 \%$ ropivacaine $(1.5$ $\left.\mathrm{ml} . \mathrm{kg}^{-1}\right)$. Exclusion criteria were patients with coagulation problems, neurological abnormalities and cardiovascular disease.

Monitoring consisted of heart rate, cardiac bullae, non-invasive systolic and diastolic blood pressure, central temperature (nasopharyngeal), $\mathrm{SpO}_{2}, \mathrm{P}_{\mathrm{ET}} \mathrm{CO}_{2}$ and neuromuscular transmission (TOF stimulation). All patients were hydrated with 10 to $15 \mathrm{ml} \cdot \mathrm{kg}^{-1} \cdot \mathrm{h}^{-1}$ lactated Ringer's and $10 \%$ glucose solution was administered at a rate of $5 \mathrm{mg} \cdot \mathrm{kg}^{-1} \cdot \mathrm{min}^{-1}$.

General anesthesia was induced with propofol ( 3 to 5 $\left.\mathrm{mg} \cdot \mathrm{kg}^{-1}\right)$, preceded by intravenous lidocaine $\left(1.5 \mathrm{mg} \cdot \mathrm{kg}^{-1}\right)$. Propofol (1\%) was slowly injected (approximately 0.1 $\left.\mathrm{mg} \cdot \mathrm{kg}^{-1} \cdot \mathrm{min}^{-1}\right)$ until loss of eyelid reflex. Muscle relaxation was obtained with atracurium $\left(0.5 \mathrm{mg} \cdot \mathrm{kg}^{-1}\right)$. Additional atracurium doses $\left(0.1 \mathrm{mg} \cdot \mathrm{kg}^{-1}\right)$ were administered when needed.

An epidural thoracic catheter was placed after anesthetic induction. Epidural puncture was performed with an $18 \mathrm{G}$ needle in $L_{5}-T_{12}$ in children above 3 months of age or weighing more than $7 \mathrm{~kg}$, and caudally and trans-sacrally in the remaining children. A catheter (20G) was introduced in the epidural space in the cephalad direction in a distance calculated for the tip to reach the thoracic region $\left(T_{7}-T_{8}\right)$. Ropivacaine was injected via catheter at an approximate rate of 0.2 $\mathrm{ml} . \mathrm{kg}^{-1} \cdot \mathrm{min}^{-1}$ with the patient in the supine position, approximately 30 degrees head-down. After $0.2 \mathrm{ml} \mathrm{kg}^{-1}$ ropivacaine, norepinephrine $(1: 200,000)$ was added for test dose.

General anesthesia was maintained with 20 and 10 $\mathrm{mg} \cdot \mathrm{kg}^{-1} \cdot \mathrm{h}^{-1}$ propofol in the Propofol and Sufentanil groups, 
respectively. Infusion rate was adjusted at 5-minute intervals to maintain blood pressure in approximately $20 \%$ of pre-induction values. Bolus 0.5 to $1 \mathrm{mg} \cdot \mathrm{kg}^{-1}$ propofol would be administered in case of inadequate anesthesia (increased muscle tone, blood pressure, heart and respiratory rate). Propofol infusion was withdrawn 10 to 15 minutes before estimated surgery completion.

Patients were mechanically ventilated to maintain $\mathrm{P}_{\mathrm{ET}} \mathrm{CO}_{2}$ between 30 and $35 \mathrm{mmHg}$ and $\mathrm{SpO}_{2}$ above $95 \%$ using a mixture of air and oxygen through a Bain system. After posterior aponeurosis closing, spontaneous ventilation would be allowed if patients could maintain $\mathrm{P}_{\mathrm{ET}} \mathrm{CO}_{2}$ below $55 \mathrm{mmHg}$ and $\mathrm{SpO}_{2}$ above $95 \%$ with $170 \mathrm{ml} \cdot \mathrm{kg}^{-1} \cdot \mathrm{min}^{-1}$ gas flow and 3 to 5 $\mathrm{cmH}_{2} \mathrm{O}$ CPAP through the Jackson-Rees system. Residual neuromuscular block was antagonized at surgery completion with atropine $\left(25 \mu \mathrm{g} \cdot \mathrm{kg}^{-1}\right)$ and neostigmine $\left(50 \mu \mathrm{g} \cdot \mathrm{kg}^{-1}\right)$. Patients were extubated in the operating room when intensively reacting to tracheal tube, showing normal respiratory pattern, opening their eyes and moving the four limbs. Intravenous naloxone (5 to $10 \mu \mathrm{g} \cdot \mathrm{kg}^{-1}$ ) would be administered if patients had respiratory depression (respiratory rate below $14 \mathrm{bpm}$ ) and myosis 5 minutes after surgery completion.

Patients were referred to PACU when reaching $\geq 8$ in the modified Aldrete-Kroulik postanesthetic recovery score ${ }^{16}$ and $\geq 3$ in the five score sedation scale ${ }^{17}$. Sedation and anesthetic recovery, blood pressure and respiratory rate were monitored in the PACU after 15, 30 and 60 minutes, and every hour from then on. $\mathrm{SpO}_{2}$ and heart rate were continuously monitored. Pain was treated with epidural morphine $\left(30 \mu \mathrm{g} \cdot \mathrm{kg}^{-1}\right)$ plus ketoprofen (intravenous $2 \mathrm{mg}^{\mathrm{kg}} \mathrm{kg}^{-1}$ every 8 hours) and intravenous tramadol ( $\left.1 \mathrm{mg} \cdot \mathrm{kg}^{-1}\right)$, used for analgesia when patients referred 3 in a 3-grade pain scale ( 1 = no pain or asleep; 2 = weak pain (crying that could be comforted); $3=$ moderate to severe pain requiring analgesics (crying or agitation unable to be comforted or pain complaint, or parents believing the child was in pain). Complications, such as respiratory depression $(\mathrm{RR}<14 \mathrm{bpm})$, nausea and vomiting were recorded during 24 hours.

Non-parametric data were analyzed by Mann-Whitney test and frequency data by Chi-square test. Level of significance was $p<0.05$.

\section{RESULTS}

Although having selected 26 children, 2 patients in each group were excluded from the study due to high resistance to epidural catheter injection.

There were no significant differences in demographics data and surgery duration between groups (Table I).

Propofol induction doses were $4.1 \pm 0.6$ and $4.5 \pm 0.7 \mathrm{mg} . \mathrm{kg}^{-1}$ in the Propofol and Sufentanil groups, respectively. Propofol infusion rate was significantly slower in the sufentanil group during 100 minutes (visceral stage) after beginning of surgery (Figure 1). Systolic blood pressure was maintained around $20 \%$ of values immediately before general anesthesia in both groups; however, heart rate went above $20 \%$ in the
Table I - Demographics Data, Surgery Duration and Puncture Site

\begin{tabular}{lcc}
\hline & $\begin{array}{c}\text { Sufentanil } \\
(\mathrm{n}=11)\end{array}$ & $\begin{array}{c}\text { Propofol } \\
(\mathrm{n}=11)\end{array}$ \\
\hline Age (months) * & $14 \pm 13.8$ & $13.7 \pm 12.6$ \\
Weight $(\mathrm{kg}){ }^{*}$ & $7.7 \pm 8.2$ & $5.8 \pm 6.3$ \\
Gender (M/F) & $7 / 4$ & $5 / 6$ \\
Surgery Duration (min) * & $103.7 \pm 14$ & $112.4 \pm 22.3$ \\
Puncture Site (number of cases) & & \\
$\quad$ Lumbar & 7 & 8 \\
$\quad$ Caudal & 4 & 3 \\
\hline
\end{tabular}

${ }^{*}$ Values expressed in Mean \pm SD

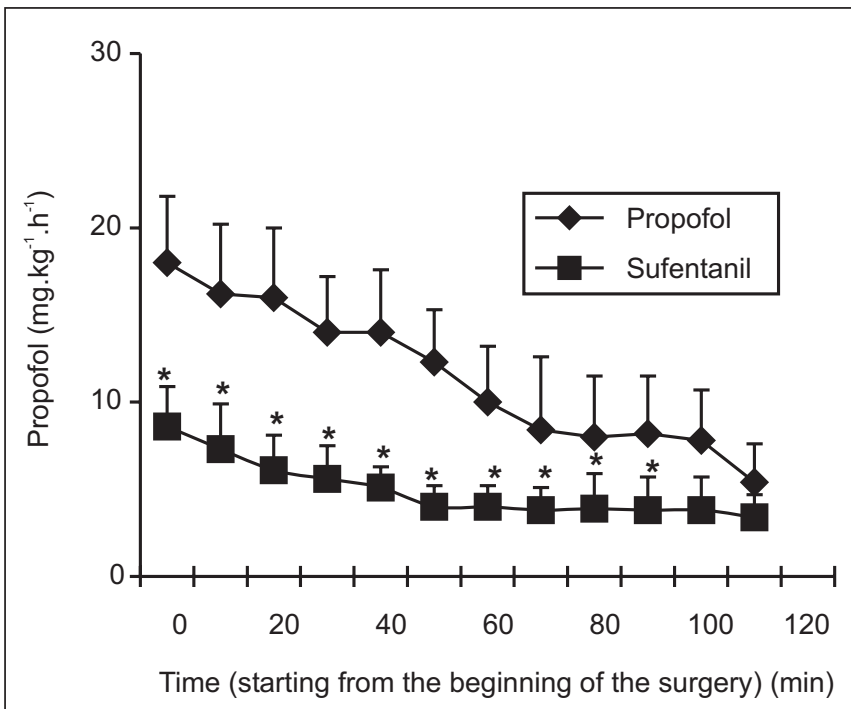

Figure 1 - Propofol Infusion Rate (Mean \pm SD)

$$
{ }^{*} p<0.05
$$

propofol group and remained constant or with a decreasing trend in the sufentanil group. Significantly more Propofol group patients needed bolus during the visceral stage ( 6 and 1 patients of Propofol and Sufentanil groups, respectively), especially when stomach and esophagus were retracted. Both groups needed 1 to 2 boluses before surgery completion to inhibit head movements during nasogastric and tracheal tube manipulation. No additional atracurium dose was needed in any patient. Two Sufentanil group patients developed transient arterial hypotension with return to normal values 5 minutes after propofol infusion withdrawal.

Spontaneous ventilation after posterior aponeurosis closing was possible in all Propofol group patients and only in 1 Sufentanil group patient. All patients were extubated in the operating room. A significantly longer time was needed for extubation and PACU referral of Sufentanil group patients as compared to Propofol group patients (Figure 2). Except for the level of sedation, which was significantly deeper in the 


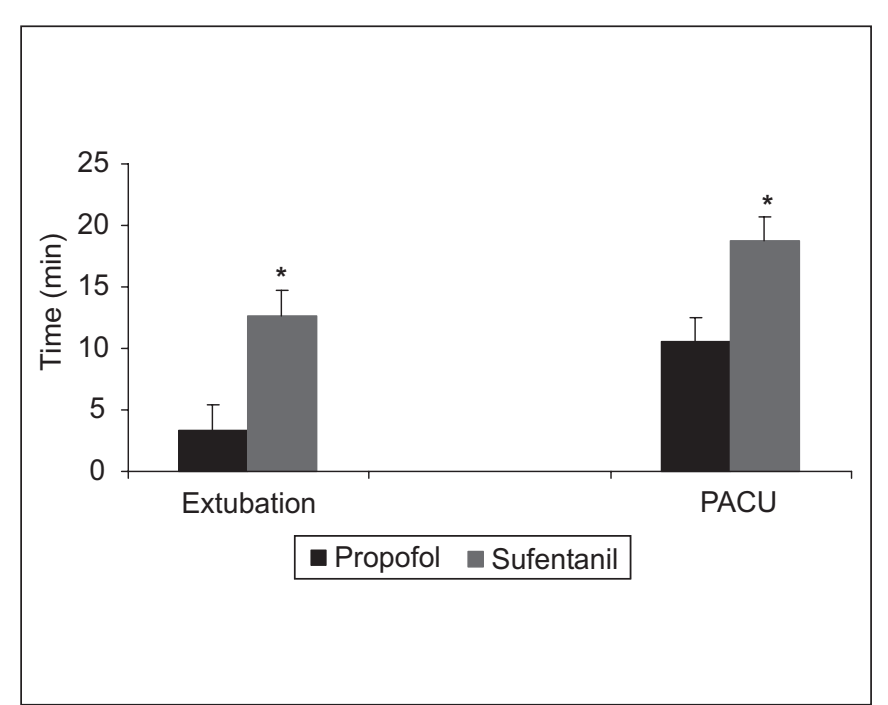

Figure 2 - Time for Extubation and PACU referral after Surgery Completion (Mean \pm SD)

$* p<0.05$

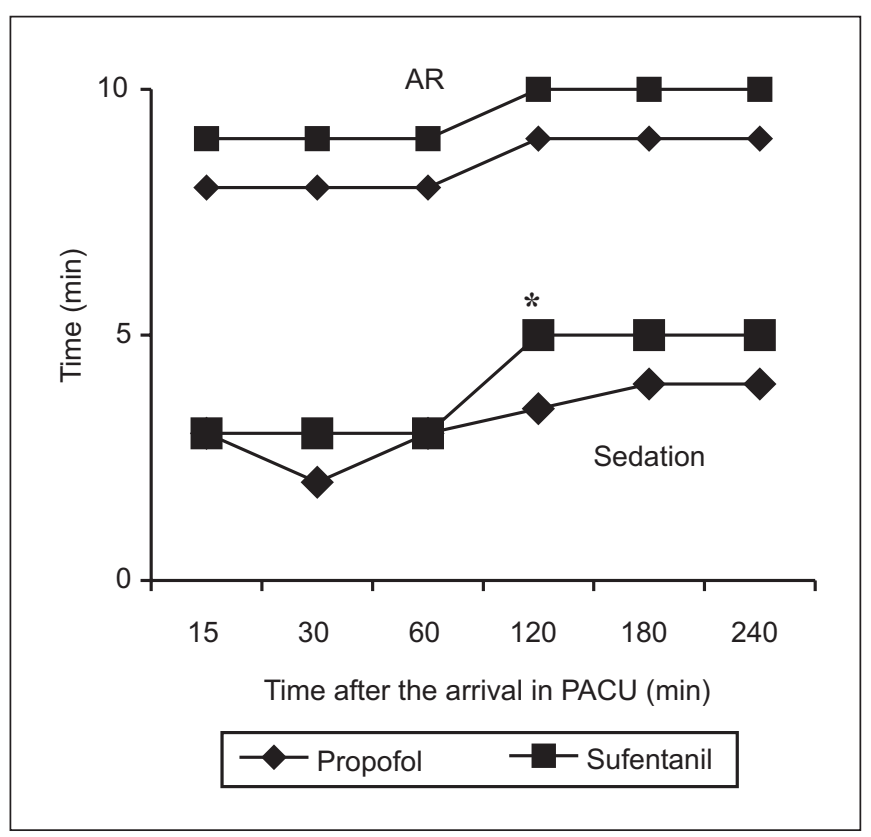

Figure 3 - Anesthetic Recovery and Sedation in PACU (Mean \pm SD) ${ }^{*} p<0.05$

Propofol group, other recovery characteristics were similar for both groups (Figure 3). Naloxone was administered to 5 Sufentanil group patients. All patients met discharge criteria (recovery score $\geq 8 \mathrm{~m}$ sedation score $\geq 3$, no complaints, pain or behavior suggesting pain, nausea, vomiting and motor block signs) 3 hours after PACU arrival. Arterial hypotension, bradycardia, arrhythmia, airway obstruction, respiratory depression or lower limb motor block signs were not observed in both groups. No patient needed analgesics in the PACU.

\section{DISCUSSION}

Both techniques used in this study may be considered total intravenous anesthesia, since after epidural ropivacaine injection only intravenous anesthetics were used. The addition of sufentanil to propofol infusion has delayed extubation and PACU discharge. However, it allowed a faster recovery, especially due to the possibility of propofol infusion at a slower rate. There were no airway obstruction, laryngospasm, cardiopulmonary depression, vomiting and motor block in the immediate postoperative period.

Propofol infusion rates needed in our study were close to those recommended by Mc Farlan et al. ${ }^{18}$, which were adequate to children between 3 and 11 years of age, but not to neonates and infants, who needed even higher doses. High propofol rate was necessary to control hemodynamic response to invasive procedures, such as tracheal intubation, epidural puncture and central vein catheterization. This study has shown that infusion rate may be decreased in at least $50 \%$ with the constant use of sufentanil $\left(1 \mu \mathrm{g} \cdot \mathrm{kg}^{-1}\right)$. Based on propofol and fentanyl requirements, a different study has concluded that abdominal traction and peritoneal incision are the most intense stimulations during surgery ${ }^{15}$, and it is possible that the same holds true for stomach and diaphragm traction. There were only 2 cases of transient arterial hypotension. Our study is in line with the observation that propofol is well tolerated by children with preserved cardiac function and that blood pressure only decreases during induction, being more frequent in older children and adolescents. Our study has not recorded clinically significant heart rate decreases and cardiac rhythm changes with the association of sufentanil ${ }^{10,19}$.

Fast recovery after prolonged propofol infusion, generally described ${ }^{20}$, was possible in patients receiving sufentanil. However, there were difficulties in returning to spontaneous ventilation in 5 Sufentanil group patients, who needed reversion with naloxone. As a consequence, time for extubation and referral to PACU was longer in the Sufentanil group. Prolonged sedation in the Propofol group is a result of prolonged infusion at higher rate as compared to the Sufentanil group.

Local anesthetic plasma levels and their systemic effects are directly related to dose, regardless of solution volumes ${ }^{21}$. For caudal or lumbar epidural anesthesia, recommended bupivacaine doses vary from 2.5 to $5 \mathrm{mg} \cdot \mathrm{kg}^{-1}$, and higher doses should be administered with epinephrine ${ }^{21,22}$.

We have used ropivaicaine $\left(3 \mathrm{mg} \cdot \mathrm{kg}^{-1}\right)$ and the first 0.2 $\mathrm{ml} . \mathrm{kg}^{-1}$ were administered with epinephrine $\left(5 \mu \mathrm{g} . \mathrm{kg}^{-1}\right)$ for detection of accidental intravascular injection. Ropivacaine solution at $0.2 \%$ was injected in the epidural space via catheter at a rate of $0.2 \mathrm{ml} . \mathrm{kg}^{-1} \cdot \mathrm{min}^{-1}$ aiming at avoiding abrupt pressure increase in the epidural space. Considering that ropivacaine has systemic toxicity and slower systemic absorption (vasoconstricting property) as compared to bupivacaine, and that an experimental study has shown that there is more resistance to ropivacaine's toxicity in young rats as compared to adult rats ${ }^{17,23}$, higher ropivaicane doses as compared to bupivacaine may be used. As a conse- 
quence, more extensive and prolonged epidural block may be safely reached. Nevertheless, several factors increase the risk for local anesthetic systemic toxicity in children as compared to adults ${ }^{24}$. Ropivacaine in a dose of $3.75 \mathrm{mg} \cdot \mathrm{kg}^{-1}$ reaches plasma peak concentration ( $\mathrm{T}_{\mathrm{MAX}}$ ) approximately 30 minutes after caudal administration and is maintained stable for 3 hours ${ }^{7}$. Highest plasma concentration was $2.2 \mu \mathrm{g}$ and no neurological effect was observed. In our study, there has been no cardiac or neurological toxicity. It is believed that 2 to $3 \mu \mathrm{g} \cdot \mathrm{ml}^{-1}$ would be the threshold for central nervous system toxicity (seizures) induced by bupivacaine ${ }^{7,25}$. It has to be admitted, however, that the actual risk for adverse neurological events or other possible adverse effects could have been masked by high propofol infusion rate and its prolonged postoperative effects.

There were no upper limb motor block, which is in line with the motor/sensory block profile described by König et al. ${ }^{7}$. Despite the high volume $\left(1.5 \mathrm{ml} . \mathrm{kg}^{-1}\right)$ injected in the thoracic epidural space, no child developed arterial hypotension (associated to epidural block), bradycardia or Horner's syndrome. Several studies have shown the proven hemodynamic stability when epidural block is associated to general anesthesia in children below 8 years of age, especially until 4 years of age. Early extubation and low incidence of adverse events in anesthetic recovery are also characteristics of the association of epidural block and general anesthesia in children ${ }^{1,2}$.

In conclusion, in our study, thoracic epidural anesthesia with $0.2 \%$ ropivacaine $\left(1.5 \mathrm{ml} \mathrm{kg}^{-1}\right)$ associated to general anesthesia with propofol or propofol plus sufentanil has promoted a safe anesthesia. The association of sufentanil has considerably decreased propofol consumption and the level of sedation in the PACU. However, time to return to spontaneous ventilation and, as a consequence, time for extubation and PACU referral may be prolonged.

\section{REFERÊNCIAS - REFERENCES}

01. Peterson KL, De Campli WM, Pike NA et al - A report of two hundred twenty cases of regional anesthesia in pediatric cardiac surgery. Anesth Analg, 2000;90:1014-1019.

02. Hammer GR, Neos K, Macario A-A retrospective examination of regional plus general anesthesia in children undergoing heart surgery. Anesth Analg, 2000;90:1020-1024.

03. Knidsen K, Becman-Suurkula M, Blomberg S et al - Central and cardiovascular effects during infusion of ropivacaine and placebo. Br J Anaesth, 1997;78:507-514.

04. Conceição MJ, Coelho L, Khalil M - Ropivacaine $0.25 \%$ compared with bupivacaine $0.25 \%$ by caudal route. Paed Anaesth, 1999;9:229-233.

05. Ivani G, Lampugnani E, De Negri P - Ropivacaine vs bupivacaine in major surgery in infants. Can J Anaesth, 1999;46:467-469.

06. Hickey R, Blanchard J, Hoffman J et al - Plasma concentrations of ropivacaine given with or without epinephrine for brachial plexus block. Can J Anaesth, 1990;37:878-882.

07. Konig H, Krenn CG, Glaser C et al - A dose-response of caudal ropivacaine in children. Anesthesiology, 1999;90:1339-1344.

Revista Brasileira de Anestesiologia

Vol. 53, № 2, Março - Abril, 2003
08. Hannallah RS, Baker SB, Casey W - Propofol dose and induction characteristics in unpremedicated children. Anesthesiology, 1991;74:217:219.

09. Aun CST, Sung RYT, O'Meara ME - Cardiovascular effects of i.v. induction in children: comparison between propofol and thiopentone. Br J Anaesth, 1993;70:647-653.

10. Williams GL, Jones TK, Hanson KA et al - The hemodynamic effects of propofol in children with congenital heart disease. Anesth Analg, 1999;89:1411-1461.

11. Westren $P$ - Induction dose of propofol in infants 1-6 months of age and children 10-16 years of age. Anesthesiology, 1991;74:455-458.

12. Browne BL, Prys-Roberts C, Wolf AR - Propofol and alfentanil in children, infusion technique and dose requirement for total i.v. anaesthesia. Br J Anaesth, 1992;69:570-576.

13. Doyle D, McFadzean W, Morton NS - IV anaesthesia with propofol using target-controlled system: comparison with inhalation anaesthesia for general surgical procedures in children. Br J Anaesth, 1993;70:542-545

14. Braithwaite P, Dowson S, Entes AH - Propofol and fentanyl infusions for cardiac surgery in children. J Cardiothor Vasc Anesth, 1990;4:126-131.

15. Kazama T, leda K, Morita K - Reduction by fentanyl of the Cp50 of propofol and hemodynamic responses to various noxious stimuli. Anesthesiology, 1997;87:213-227.

16. Ates $\mathrm{Y}$, Ünal N, Cuhruk $\mathrm{H}$ et al - Postoperative analgesia in children using a preemptive retrobulbar block and local anesthetic infiltration in strabismus surgery. Reg Anesth Pain Med, 1998;23:569-574.

17. Yaster M, Billet C - Intravenous Patient Controlled Analgesia, em: Yaster M, Krane EJ, Kaplan RF et al - Pediatric Pain Management and Sedation Handbook, New York, Mosby-Year Book Inc, 1997;89-111.

18. Mc Farlan CS, Anderson B, Short T - The use of propofol infusion in paediatric anaesthesia: a practical guide. Paed Anaesth, 1999;9:209-216.

19. Hertzog GH, Campbell JK, Dalton H et al - Propofol anesthesia for invasive procedures in ambulatory and hospitalized children: experience in the paediatric intensive care unit. Paediatrics, 1999;103:E30.

20. Picard V, Dumont L, Pellegrine M - Quality of recovery in children: sevoflurane versus propofol. Acta Anaesthesiol Scand, 2000;44:307-310.

21. Yaster M, Maxwell LG - Pediatric regional anesthesia. Anesthesiology, 1989;70:324-338.

22. Rowney DA, Doyle E - Epidural and subarachnoid blockade in children. Anaesthesia, 1998;53:980-1001.

23. Kohane DS, Sankar WN, Shubina M et al - Sciatic nerve blockade in infant, adolescent, and adult rats. Anesthesiology, 1998;89:1199-1208.

24. Dalens BJ, Mazut JX - Adverse effects of regional anesthesia in children. Drug Saf, 1998;19:251-268.

25. Berde CB - Toxicity of local anesthetic in infants and children. J Pediatr, 1993;22:14-20.

\section{RESUMEN}

Klamt JG, Garcia LV, Stocche RM, Reis MP - Anestesia Peridural Contínua con Ropivacaína a 0,2\% Asociada a Anestesia General para Cirugía del Abdomen Superior en Niños

Justificativa y Objetivos - Técnicas anestésicas han sido empleadas en pacientes pediátricos para varios tipos de cirugías, presentando entre otras ventajas, la analgesia 
pós-operatoria. El objetivo de este estudio fue evaluar el ritmo de infusión de propofol y la recuperación pós-anestésica de niños sometidos a cirugía abdominal alta bajo anestesia peridural torácica con ropivacaína a $0,2 \%$, asociada a anestesia general con propofol o propofol más sufentanil.

Método - Veintiséis niños ASA I, II y III, con edades entre 0 y 4 años, sometidos a cirugía abdominal alta fueron seleccionados para anestesia peridural torácica $\left(T_{7}-T_{8}\right)$ con ropivacaína a $0,2 \%\left(1,5 \mathrm{ml} . \mathrm{kg}^{-1}\right)$. Fueron divididos aleatoriamente en dos grupos: Propofol (infusión de propofol) y Sufentanil (infusión de propofol más sufentanil $1 \mu \mathrm{g} . \mathrm{kg}^{-1}$ ). Los ritmos de infusiones de propofol fueron de 20 y $10 \mathrm{mg} \cdot \mathrm{kg}^{-1} . \mathrm{h}^{-1}$ en los grupos Propofol y Sufentanil, respectivamente, ajustadas de modo a mantener la presión arterial cerca de $20 \%$ de los valores pré-inducción e interrumpidas 10 a 15 minutos antes del final estimado de la cirugía. La recuperación pós-anestésica fue evaluada a través de una escala modificada de Aldrete-Kroulik y sedación evaluada a través de una escala de 5 puntos.
Resultados - Dos niños de cada grupo fueron excluidos por problemas técnicos. El ritmo de infusión fue significativamente menor en el grupo Sufentanil en relación al grupo Propofol durante 100 minutos después del inicio de la cirugía. Los tiempos para extubación y transferencia para la sala de recuperación pós-anestésica (SRPA) fueron significativamente menores en el grupo Propofol, sin embargo, la intensidad y duración de la sedación fueron mayores en ese grupo en relación al grupo Sufentanil. Los escores de recuperación fueron similares en los dos grupos. Después de 3 horas en la SRPA, todos los pacientes habian llegado a los criterios de transferencia para las enfermarías. Hipotensión arterial transitoria fue observada en 2 pacientes del grupo Sufentanil.

Conclusiones - La combinación de la anestesia peridural torácica continua con ropivacaína a $0,2 \% \quad\left(1,5 \mathrm{ml} . \mathrm{kg}^{-1}\right)$ asociada a la infusión de propofol promueve anestesia efectiva y segura para cirugías abdominales altas en niños. El ritmo de infusión de propofol y el tiempo de sedación fueron reducidos con la adición de sufentanil. 